\title{
Simulative Study of Wavelength Division Multiplexing Fiber Bragg Grating in Nuclear Reactors Monitoring
}

\author{
Abd El-Naser A.Mohamed*, Ahmed Nabih Zaki Rashed", M. M. \\ Zaky**, Ahmed I.Elsaket***, Mohamed A.Gaheen*** \\ * Dept. of Electronics and Electrical Communications Engineering, Faculty of \\ Electronic Engineering, Menoufia University \\ ** Dept. of Engineering, Nuclear Research Center, Atomic Energy Authority \\ ${ }^{* * *}$ Dept. of Nuclear Safety and Radiological Emergencies, NCRRT.
}

(Received: 28 Mar. 2018 - Accepted: 10 Oct. 2018)

\section{Abstract}

The technologies of wavelength division multiplexing (WDM) have been theoretically studied and analyzed for multiplexing fiber Bragg grating (FBG) in a single optical fiber. This method allows a single fiber to carry many of identical FBGs, making this sensor more appropriate in the nuclear reactors. The analysis demonstrates that the multiplexing capacity can be incredibly enhance small data rates and high channel spacing. The interference effect among FBGs multi-reflections channels must be taken into account. This paper simulate WDM based FBG for a channel spacing of $0.1,0.3$, $0.5,0.8,1 \mathrm{~nm}$ Gaussian apodized FBGs at data rates of 2.5, 10, $40,100,160,250 \mathrm{~Gb} / \mathrm{s}$ respectively for nuclear applications. All simulations were performed in Optisystem software.

\section{Introduction}

A large number of transmission channels and high data rate have made of fiber optic systems which are more applicable for nuclear applications [1]. For better implementation and practical accomplishment optical fiber is required to decrease the waste material. Optical fiber networks give higher data rate than copper based systems [2]. Due to demand for high transmission capacity system the wavelength division multiplexing (WDM) is progressing. WDM optical systems are created to help numerous signals with various wavelengths to transmission as well as to increase security and integrity of data in a single fiber. For example, 
several thermocouples can be substituted by a single fiber. They are utilized for effective use of bandwidth and achieving high system capacity [1-2].

Recently, fiber Bragg grating (FBG) becomes the optical technology that mostly used for several applications such as nuclear reactors monitoring. Optical FBG are generally used as the optical sensor in a nuclear reactor [3]. In particular, FBG represents the key sensing component, since it gives us the opportunity to detect many parameters: temperature, rotation, vibration and pressure [4]. An FBG is a reflective device carved into the fiber optic cable by ultraviolet radiation. Reflected wavelength of FBG changes with the refractive index and grating length. Advantages of FBGs over other technologies such as fiber geometry, high return loss, low insertion loss, immune to electromagnetic interference and potentially low cost [5] since the WDM incredibly improves the capacity of the optical systems. FBGs have a radiation sensitivity which highly dependent on the chemical composition and photosensitization method used for fabrication [6]. The shape of gratings spectra and amplitude remained unchanged after long period of radiation for fibers without hydrogen loading, but showed only slight changes for fibers with hydrogen loading [7].

This paper deals with the concept of WDM as techniques to use the FBG sensor array with the minimum interference and detects suitable channel spacing at various data rates in nuclear sensing applications. Examine WDM multiplexing networks for 4, 8, 16 and 32 channels of FBGs sensors on a single fiber cable without interference to be applicable in nuclear sensing application such as temperature monitoring inside a nuclear reactor core and take advantage of the multiplexing capabilities of FBG temperature sensors to perform in core multipoint temperature measurements. The simulation of the spectral response and efficiency of such a multiplexer for different data rate with different channel spacing are presented. Results are discussed using Optisystem.

\section{Bragg Grating Sensors Multiplexing}

A Fiber Bragg Grating ( $\mathrm{FBG}$ ) is a periodic modulation perturbation of the effective refractive index (neff) of the core of an optical fiber. As shown in Fig.1, at the Bragg wavelength $\left(\lambda_{B}\right)$ a narrow spectral band is reflected 
back by the grating, where $\lambda_{B}$ depend upon effective refractive index $\left(n_{\text {eff }}\right)$ and grating period $(\Lambda)$ as $[8]$ :

$$
\lambda_{B}=2 n_{e f f} \Lambda
$$

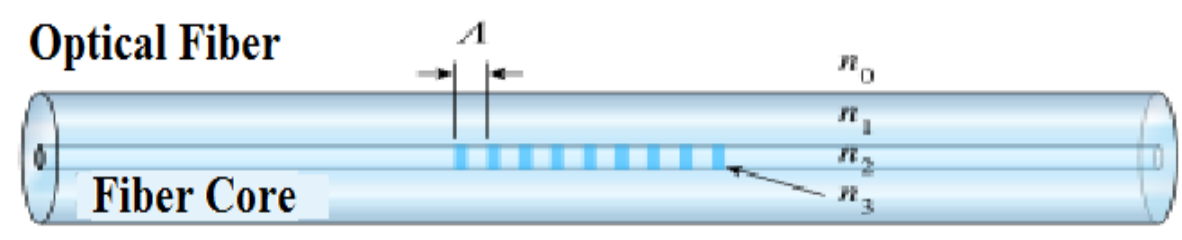

n * Core Refractive Index

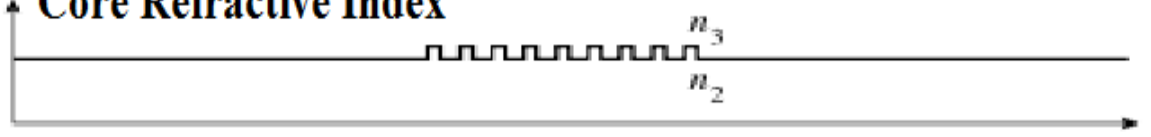

Spectral Response
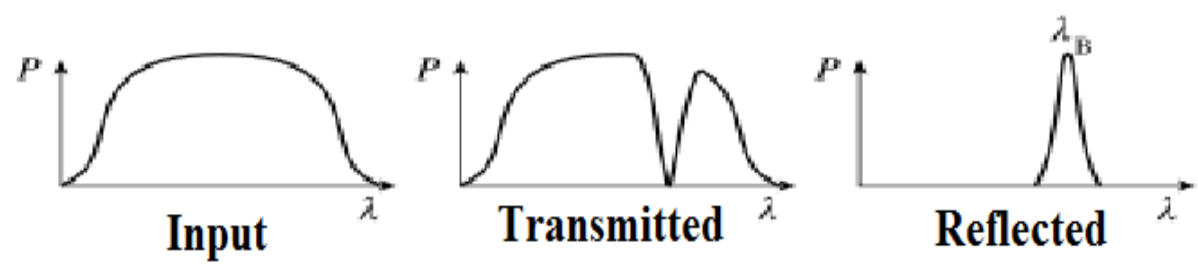

Figure1. FBG Sensing Mechanism.

The effective refractive index and the grating period between FGB planes are affected by changes in temperature or strain, also the Bragg wavelength shifted and expressed as [9]:

$\Delta \lambda_{B}=\lambda_{B}\left[\Delta T(\alpha+\xi)+\varepsilon\left(1-\frac{n^{2}{ }_{e f f}}{2}\left[p_{12}-v\left(p_{12}+p_{11}\right)\right]\right)\right.$

where the first term $\Delta T$ refer to the change in temperature, $\alpha$ is the thermal expansion coefficient for the fiber and $\xi$ is the thermo-optic coefficient. And the second term $\varepsilon$ refer to applied strain along the longitudinal axis, $\left(p_{11}, p_{12}\right)$ are the components of the fiber optic strain sensor also known as the Pockels constants of the fiber, $\mathrm{n}_{\mathrm{eff}}$ is the effective refractive index and $v$ is the Poisson's ratio. The new wavelength must be recorded. FBG have different resonant wavelengths since we can measure 
stresses and temperature at different intervals using single fiber integrated with multiplexing.

The essential advantage for FBGs is that every grating element reflects various Bragg wavelengths. Many FBGs sensors can be exposed at specific locations along a single fiber [10]. The sensors can detect the same or different physical parameters at different areas of an optical system. For WDM networks, various FBGs sensors have a specific central Bragg wavelength. The other sensors can be isolated by little nanometers. The WDM interrogation shows high resolution, stability, accuracy and flexibility with feasible numbers of sensors and comparatively low sampling rates [11-12].

As shown in Fig. 2, the designed model utilized in this article has been created by simulation programming OptiSystem. WDM is an important advance in the development history of any fiber optical communication technology. The main principle of the WDM is that the optical signals with different wavelengths are put together at first, and then coupled to optic fiber cable lines in the same fibers for transmission [13]. A multiplexer must be added at the transmitter to combine all the channels so that they can be transmitted through the optical fibers. The system consists of continuous wave (CW) laser array, wavelength division multiplexer (WDM), mach-zehnder modulator (MZM) which operating in fiber optics networks all over the world and used in an increasing number of photonics applications such as fiber optics sensors, fiber lasers systems and measurement equipment. A Mach-Zehnder modulator is used for controlling the amplitude of an optical wave. The input waveguide is split into two paths that are then recombined into an output waveguide. The two paths make up the two arms of the interferometer and the optical index modulation induced on each of them creates the intensity modulation at the output of the device as shown in Fig.3, FBG, optical spectrum analyzers and other devices. The light signals from CW lasers array are put together through the WDM and then coupled into the optical fiber. The model uses pulse generator non-return-zero (NRZ) for producing electrical data signal owing to the modulation process. The $\mathrm{NRZ}$ is used to fix the bits in the state according to the voltage changes. 


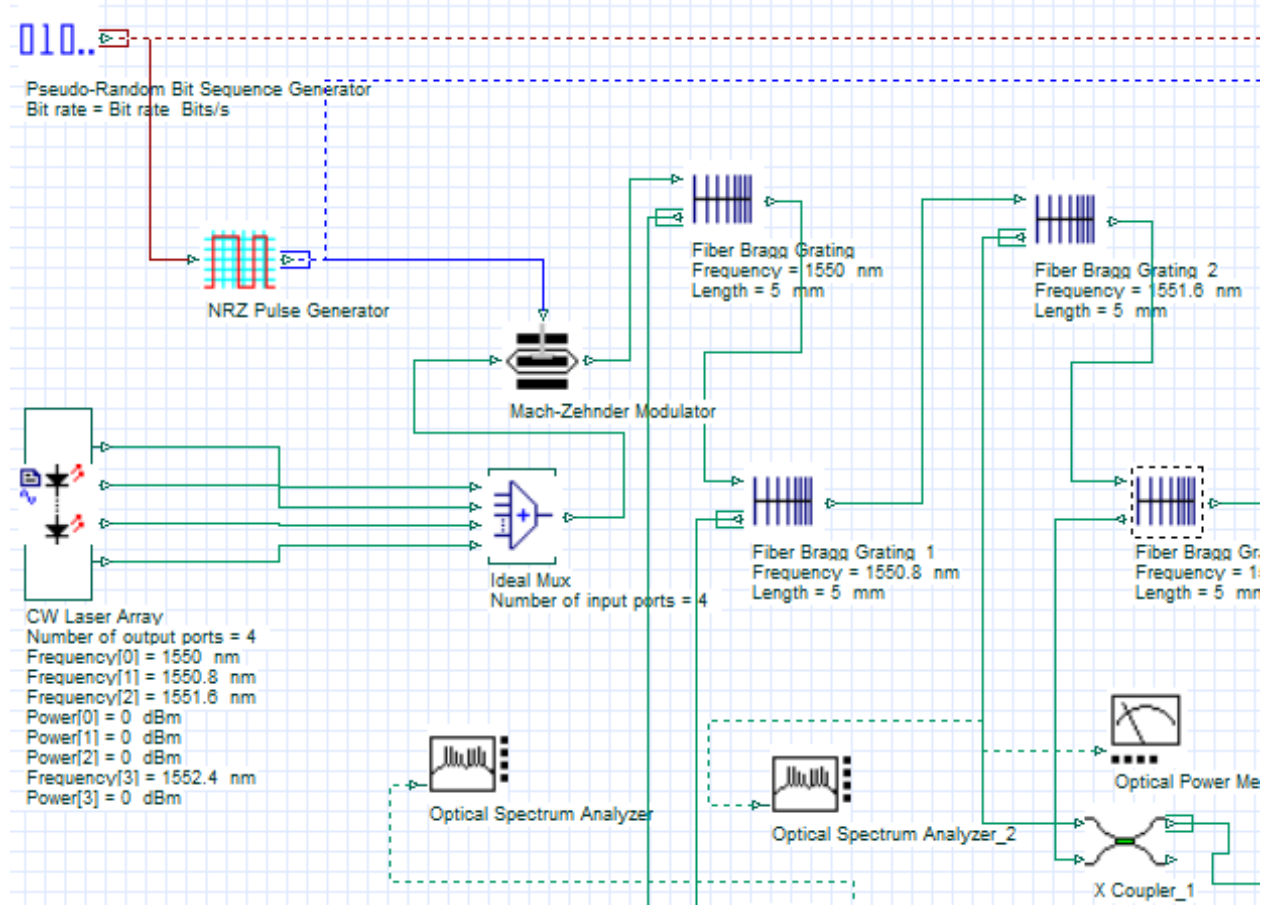

Figure 2. WDM FBG array simulation design model with optisystem software

In addition, the NRZ pulse generator has an advantage in controlling bandwidth. The 4 port continuous wave laser source is used to give the input. Pseudo Random Bit Sequence Generator (PRBS) is used to generate an arbitrary bit sequence which is given to NRZ pulse generator. This electrical signal is then modulated with the incoming optical signal using a mach-zehnder modulator (MZM). The sensing area consists of four identical FBGs connected via single mode fiber. The reflected back signals are coupled and fed into the photo detector to be detected and analyzed by the optical spectrum analyzer. 


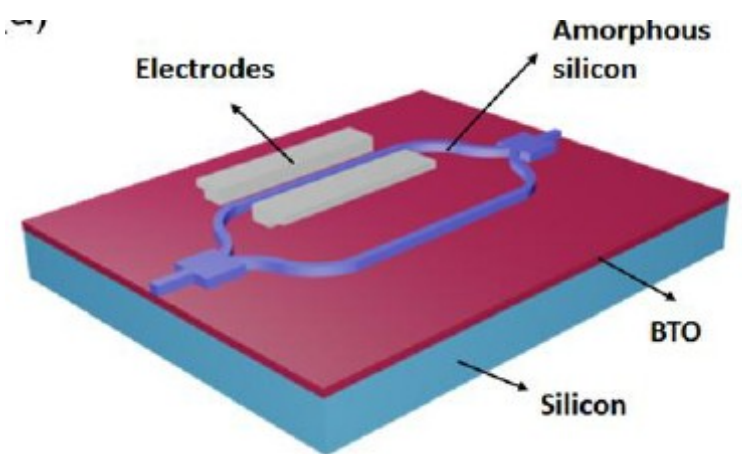

Figure 3. Schematic of the Mach-Zehnder Modulator

\subsection{Simulation result for 4-sensor array}

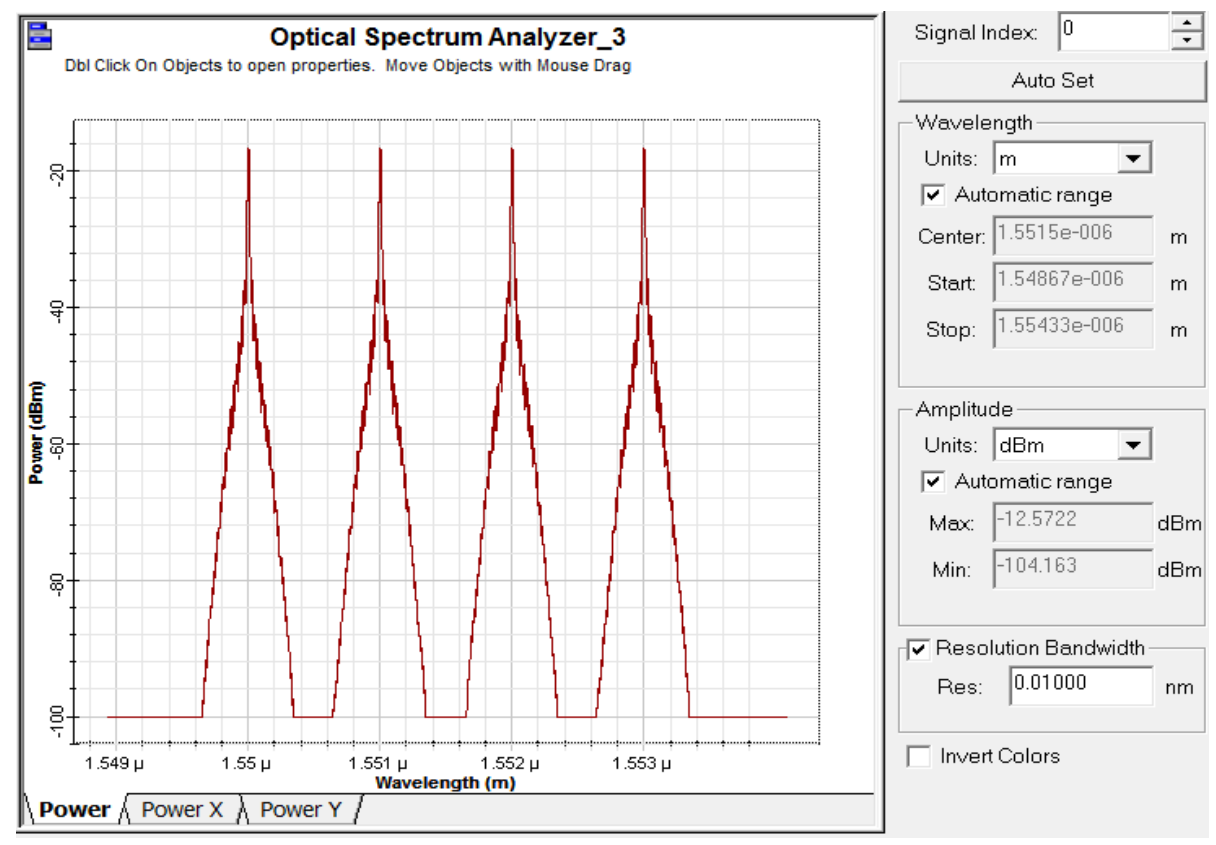

Figure 4. Reflected spectrum is simulated for the set of four sensors at channel spacing $1 \mathrm{~nm}$ at data rate $2.5 \mathrm{~Gb} / \mathrm{s}$. 
Figs. 4 and 5 show the reflected spectrum for the set of four sensors at the data rate of $2.5 \mathrm{~Gb} / \mathrm{s}$. The maximum signal amplitude is reached to $12.5722 \mathrm{dBm}$, which the minimum signal amplitude is reached to $104.163 \mathrm{dBm}$. As well as it is observed with increasing the transmission data rate to $10 \mathrm{~Gb} / \mathrm{s}$, the maximum signal amplitude is reached to -13.606 $\mathrm{dBm}$, while its minimum signal amplitude is reached to $-104.114 \mathrm{dBm}$. The interference increase as data rate increases.

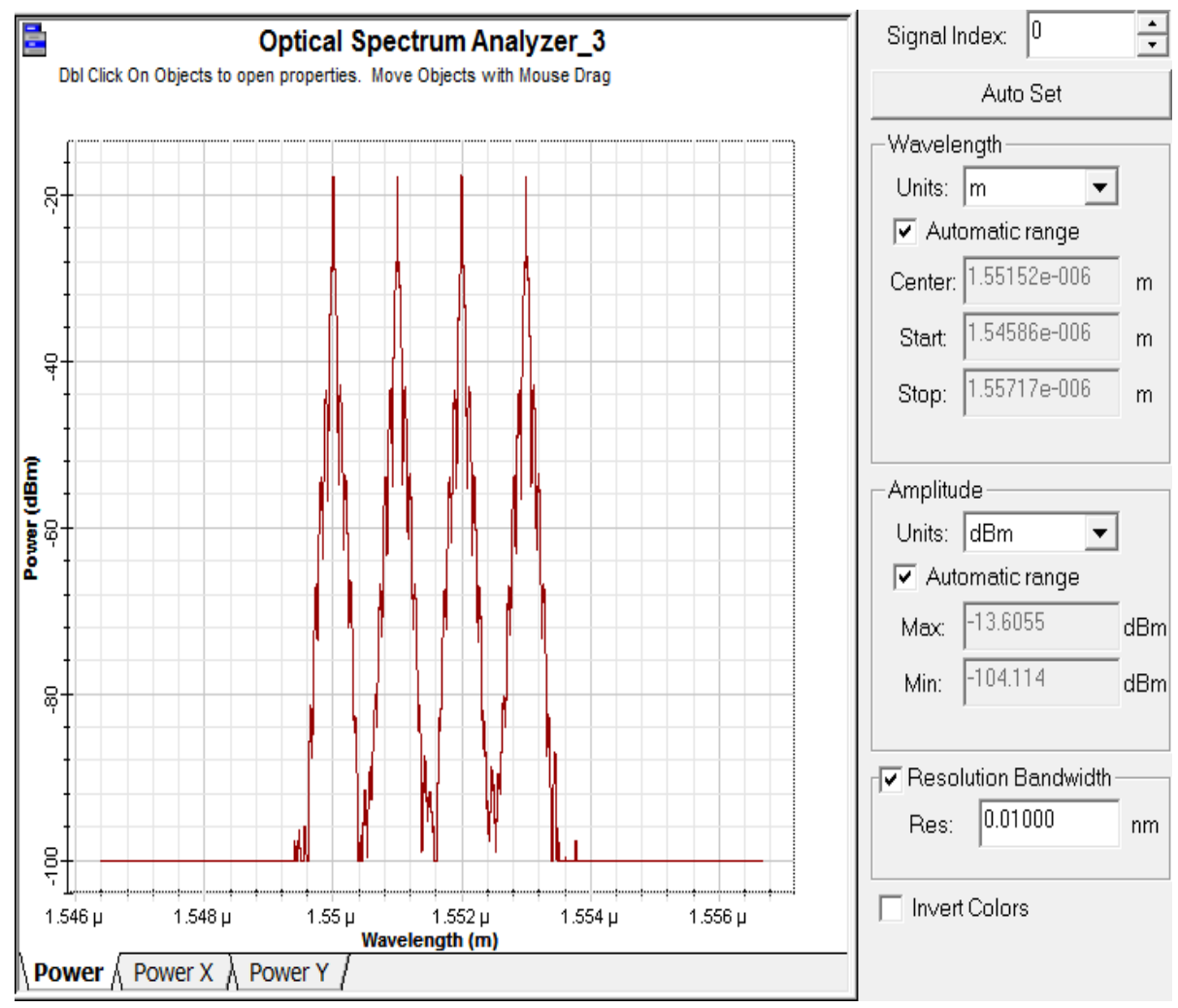

Figure 5. Reflected spectrum is simulated for the set of four sensors at channel spacing $1 \mathrm{~nm}$ at data rate $10 \mathrm{~Gb} / \mathrm{s}$. 


\subsection{Simulation result for 8-sensor array}

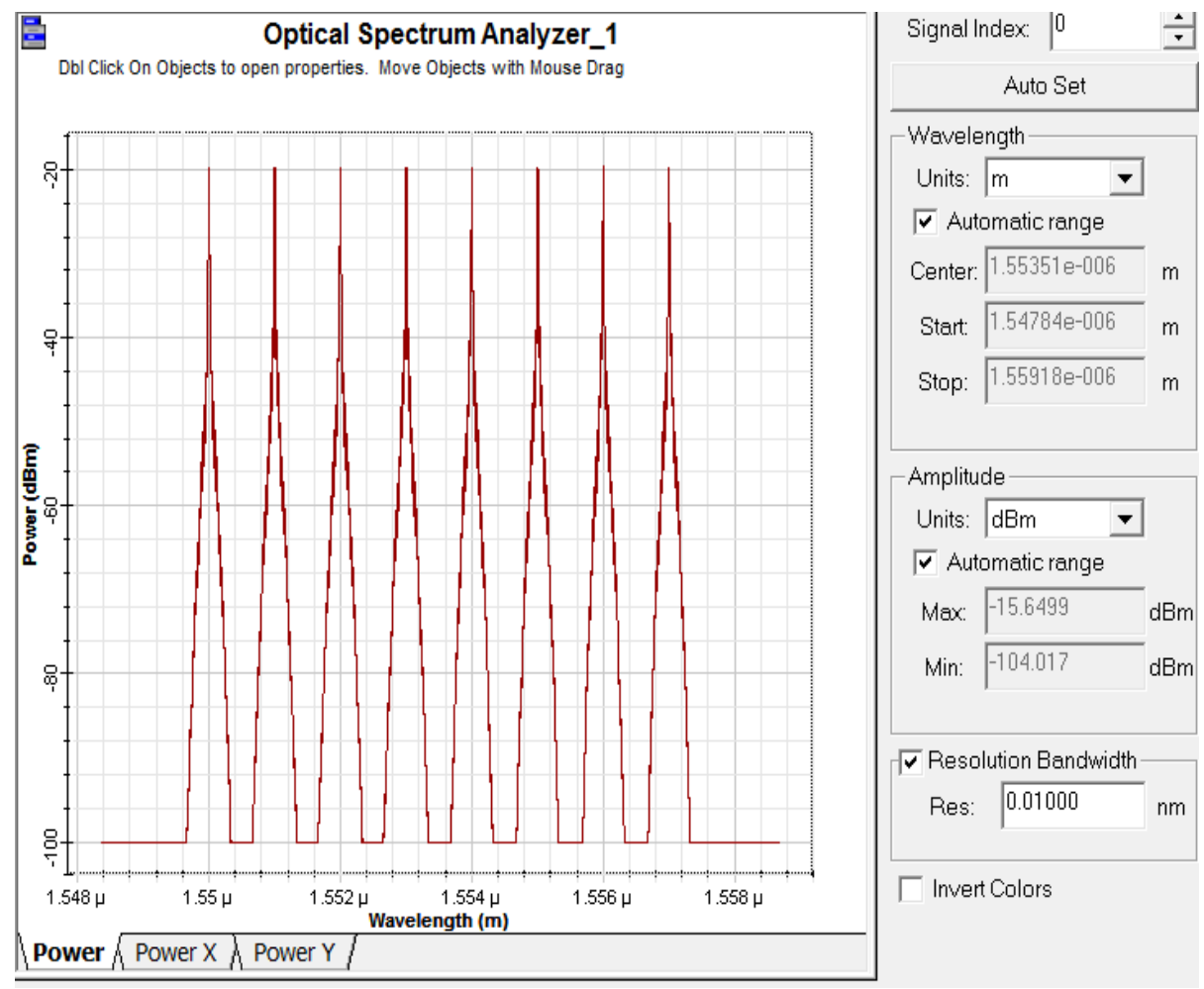

Figure 6. Reflected spectrum is simulated for the set of 8 sensors at channel spacing $1 \mathrm{~nm}$ at data rate $2.5 \mathrm{~Gb} / \mathrm{s}$.

Reflected spectrum for the set of eight sensors at a data rate of $2.5 \mathrm{~Gb} / \mathrm{s}$ shown in Figs. 6 and 7. The maximum signal amplitude is reached to $15.6499 \mathrm{dBm}$, which the minimum signal amplitude is reached to $104.017 \mathrm{dBm}$. As well as it is observed with increasing the transmission data rate to $10 \mathrm{~Gb} / \mathrm{s}$, the maximum signal amplitude is reached to -16.7096 $\mathrm{dBm}$, while its minimum signal amplitude is reached to $-103.966 \mathrm{dBm}$. Interference increase at $10 \mathrm{~Gb} / \mathrm{s}$ for eight sensor array. 

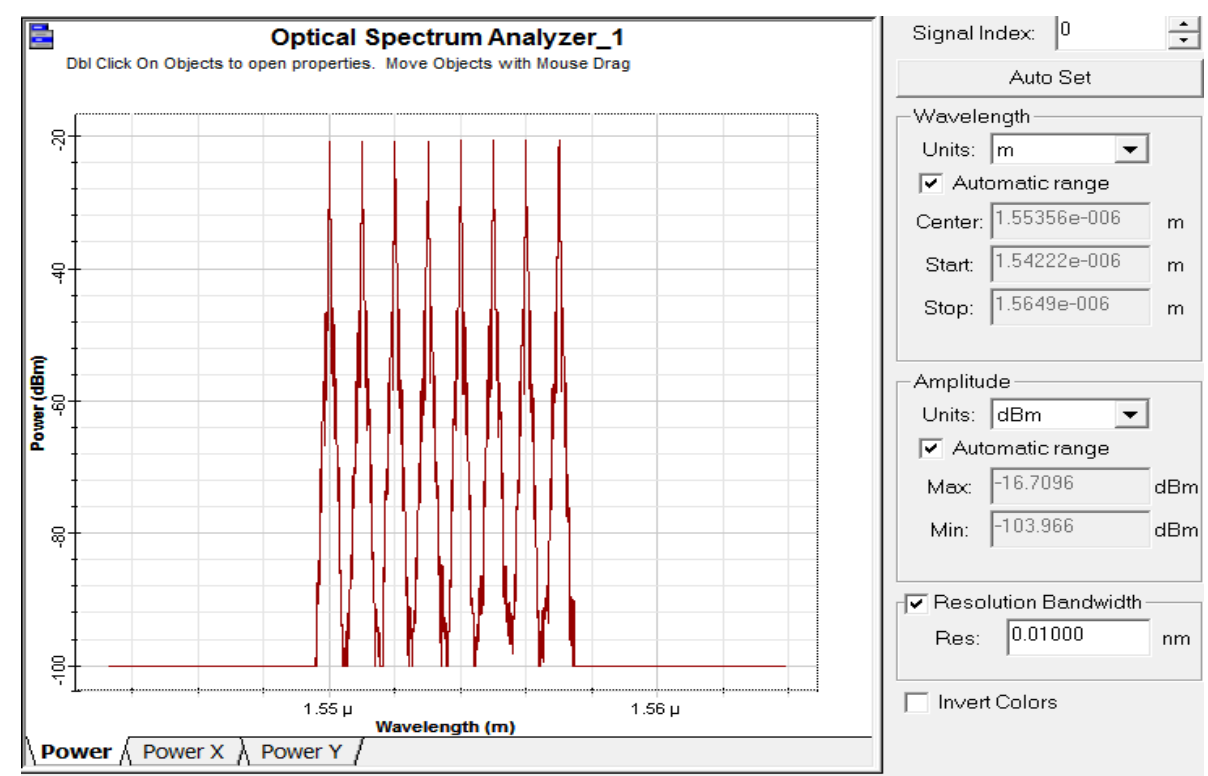

Figure 7. Reflected spectrum is simulated for the set of 8 sensors at channel spacing $1 \mathrm{~nm}$ at data rate $10 \mathrm{~Gb} / \mathrm{s}$.

\subsection{Simulation result for $\mathbf{1 6 - s e n s o r}$ array}
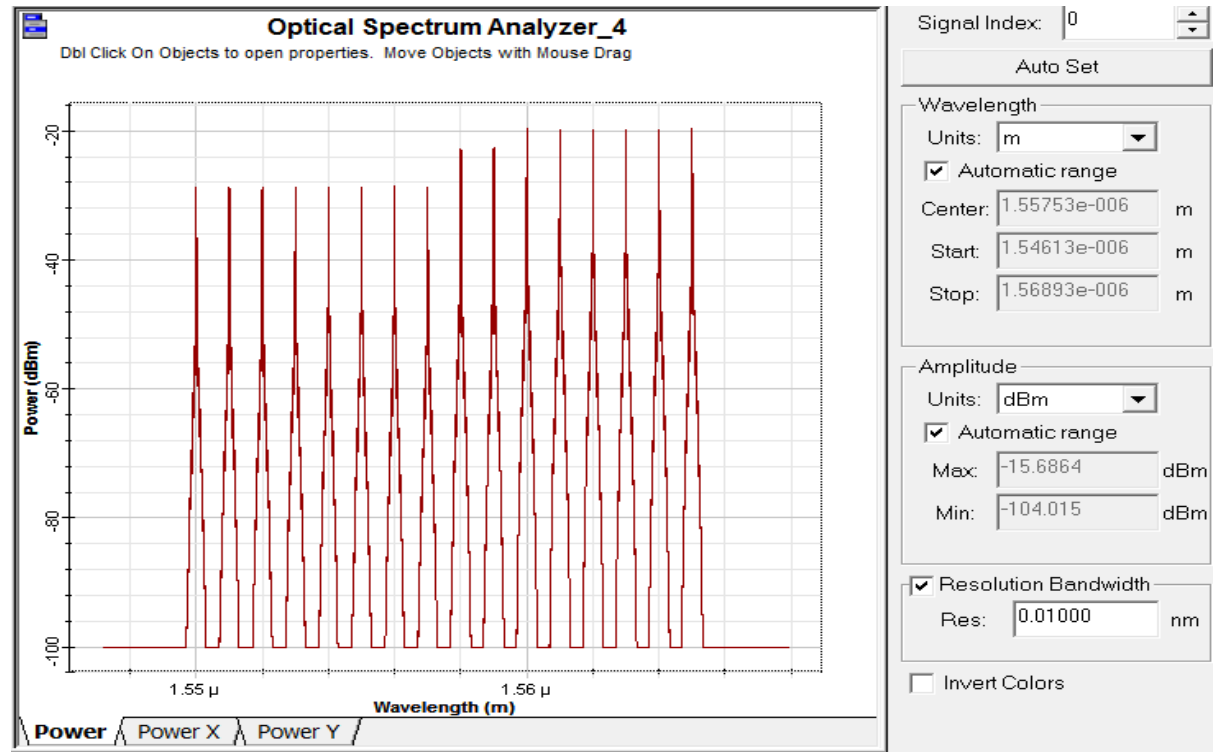

Figure 8. Reflected spectrum is simulated for the set of 16 sensors at channel spacing $1 \mathrm{~nm}$ at data rate $2.5 \mathrm{~Gb} / \mathrm{s}$. 
For the set of sixteen sensors at the data rate of $2.5 \mathrm{~Gb} / \mathrm{s}$, reflected spectrum illustrated in Figs. 8 and 9. The maximum signal amplitude is reached to $-15.6864 \mathrm{dBm}$, which the minimum signal amplitude is reached to $-104.015 \mathrm{dBm}$. As well as it is observed with increasing the transmission data rate to $10 \mathrm{~Gb} / \mathrm{s}$, the maximum signal amplitude is reached to $-16.6916 \mathrm{dBm}$, while its minimum signal amplitude is reached to $-103.967 \mathrm{dBm}$. The case of 16 sensor array at $2.5 \mathrm{~Gb} / \mathrm{s}$ is applicable for nuclear application.

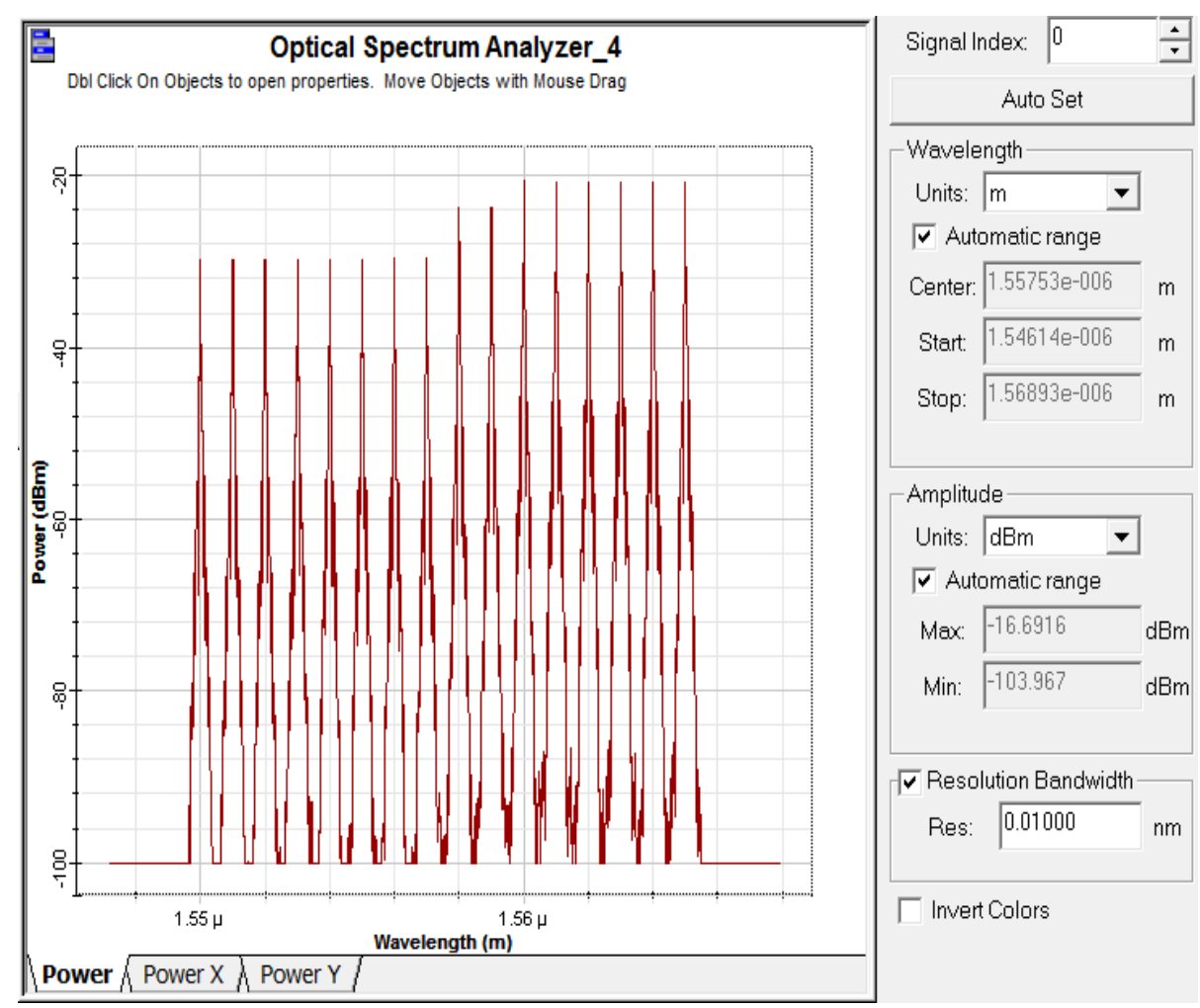

Figure 9. Reflected spectrum is simulated for the set of 16 sensors at channel spacing $1 \mathrm{~nm}$ at data rate $10 \mathrm{~Gb} / \mathrm{s}$. 


\subsection{Simulation result for 32-sensor array}
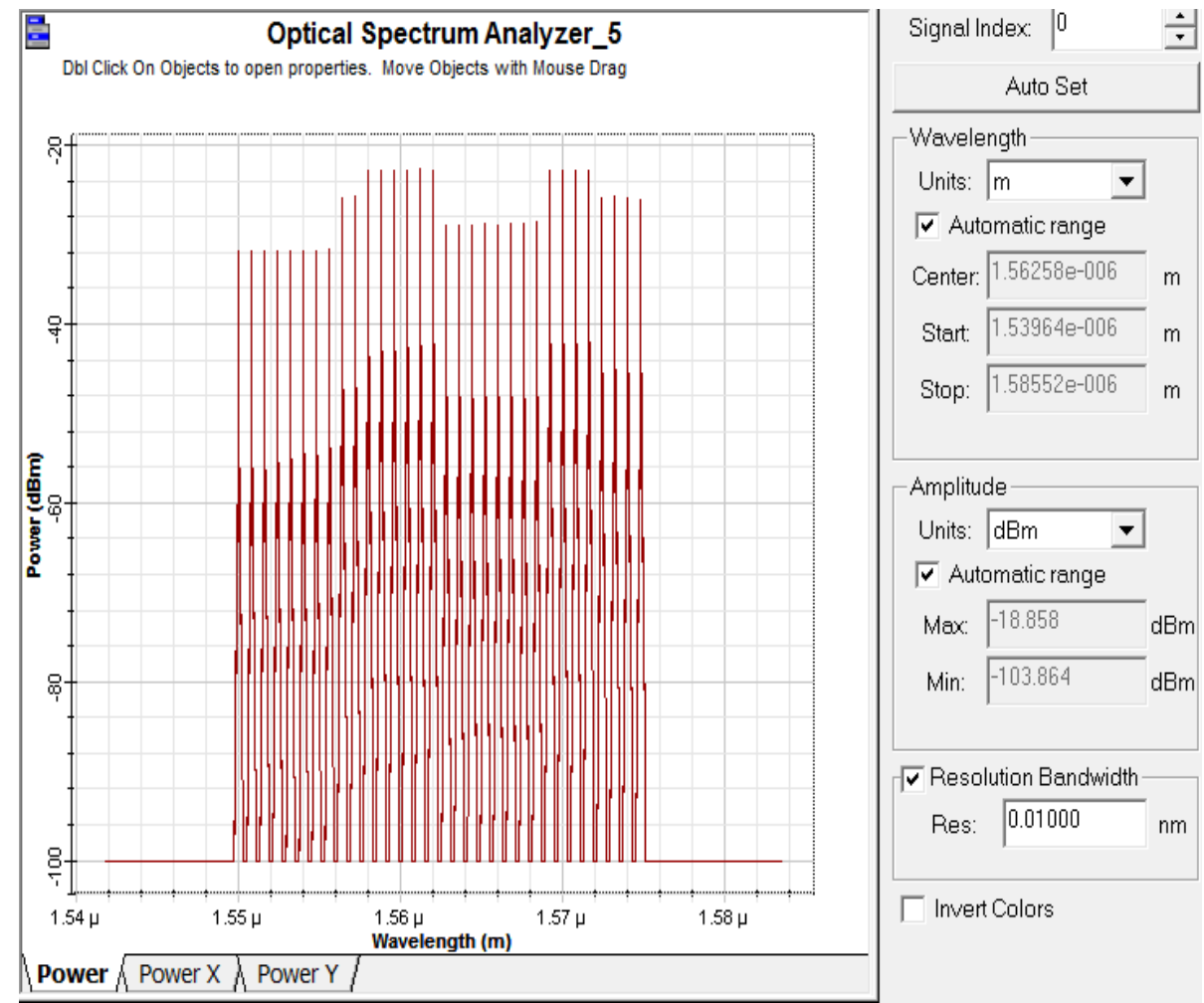

Figure 10. Reflected spectrum is simulated for the set of 32 sensors at channel spacing $1 \mathrm{~nm}$ at data rate $2.5 \mathrm{~Gb} / \mathrm{s}$.

Figs. 10 and 11 illustrate the reflected spectrum for the set of thirty two sensors at the data rate of $2.5 \mathrm{~Gb} / \mathrm{s}$. The maximum signal amplitude is reached to $-18.858 \mathrm{dBm}$, which the minimum signal amplitude is reached to $-103.864 \mathrm{dBm}$. As well as it is observed with increasing the transmission data rate to $10 \mathrm{~Gb} / \mathrm{s}$, the maximum signal amplitude is reached to $-19.9138 \mathrm{dBm}$, while its minimum signal amplitude is reached to $-103.814 \mathrm{dBm}$.. The array of $32 \mathrm{FBG}$ high interference was observed at $2.5 \mathrm{~Gb} / \mathrm{s}$ and increase with increasing data rate. 


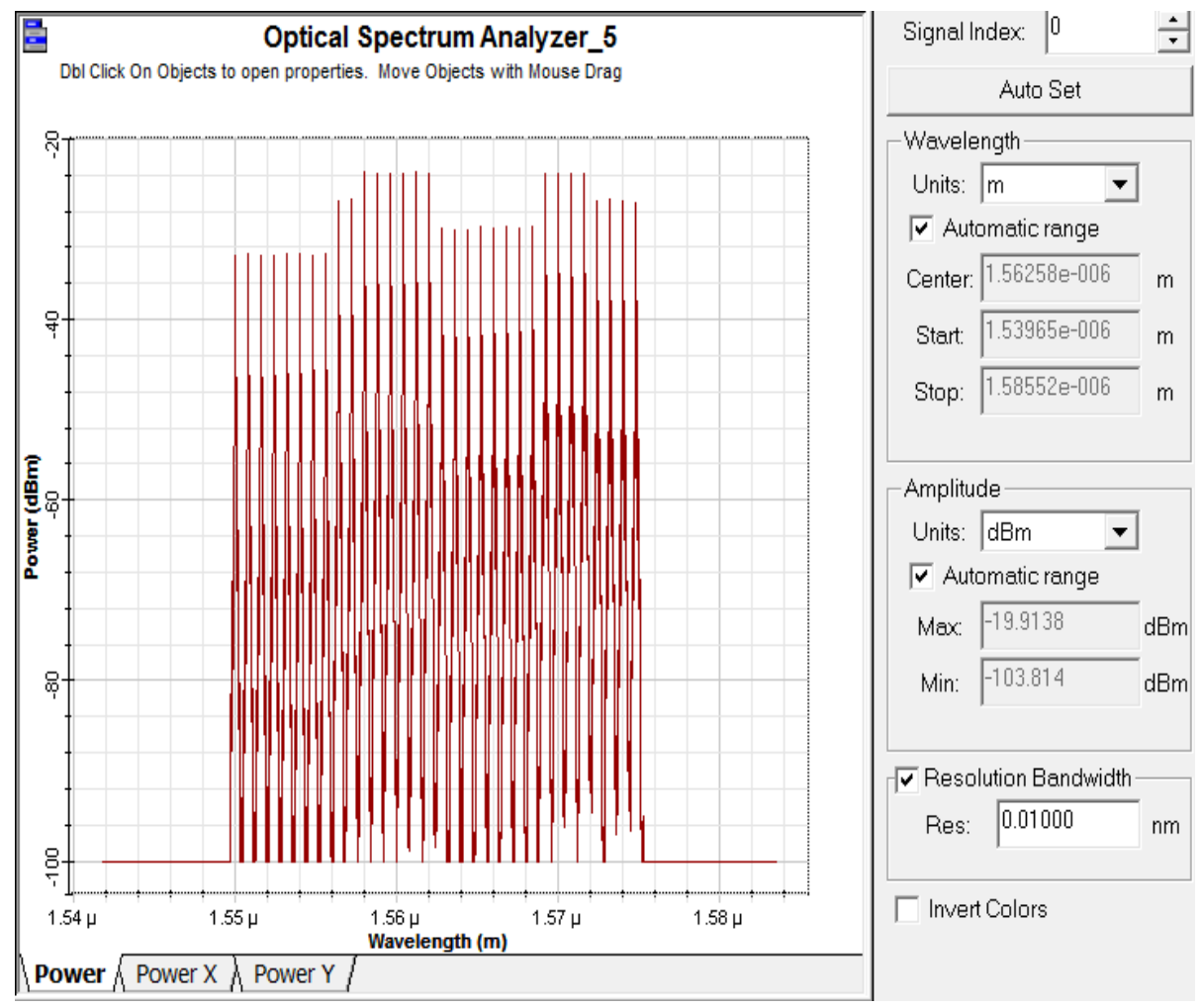

Figure 11. Reflected spectrum is simulated for the set of 32 sensors at channel spacing $1 \mathrm{~nm}$ at data rate $10 \mathrm{~Gb} / \mathrm{s}$.

Power of reflected signal is ranging between -20 and $-40 \mathrm{dBm}$ [2]. As shown in Fig.12, maximum reflected signal power ranges between -12.5 to $-19.9 \mathrm{dBm}$ and no overlap between adjacent channels when the design model operated at $1 \mathrm{~nm}$ channel spacing. Improving the capacity of the optical system can be enhanced with decreasing channel spacing as shown in Figs.12, 13. As well as the reflected power increases, reflectivity of FBG increases, which give more accurate reading [14]. An overlapping between reflected spectrums and side lobes give undesirable spectrum shown in Figs. 5, 7, 9, 11. 


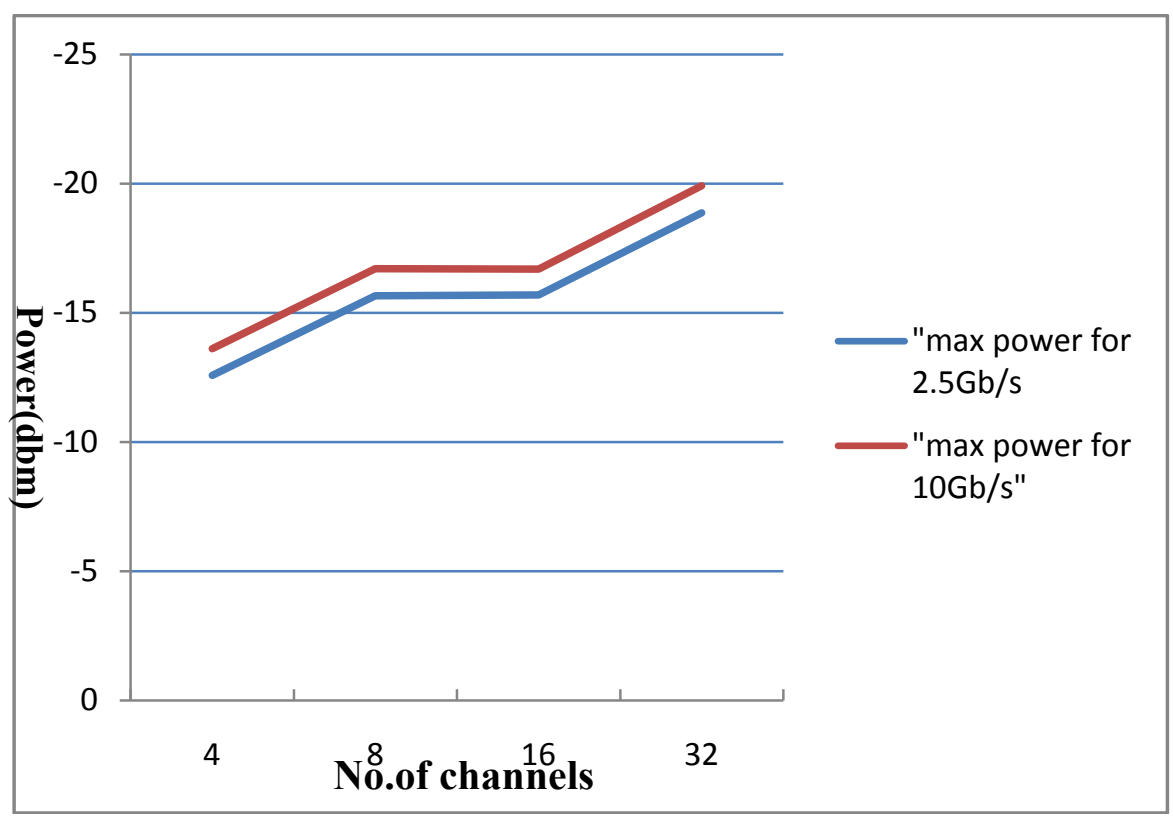

Figure 12. Relationship between maximum power and number of channels

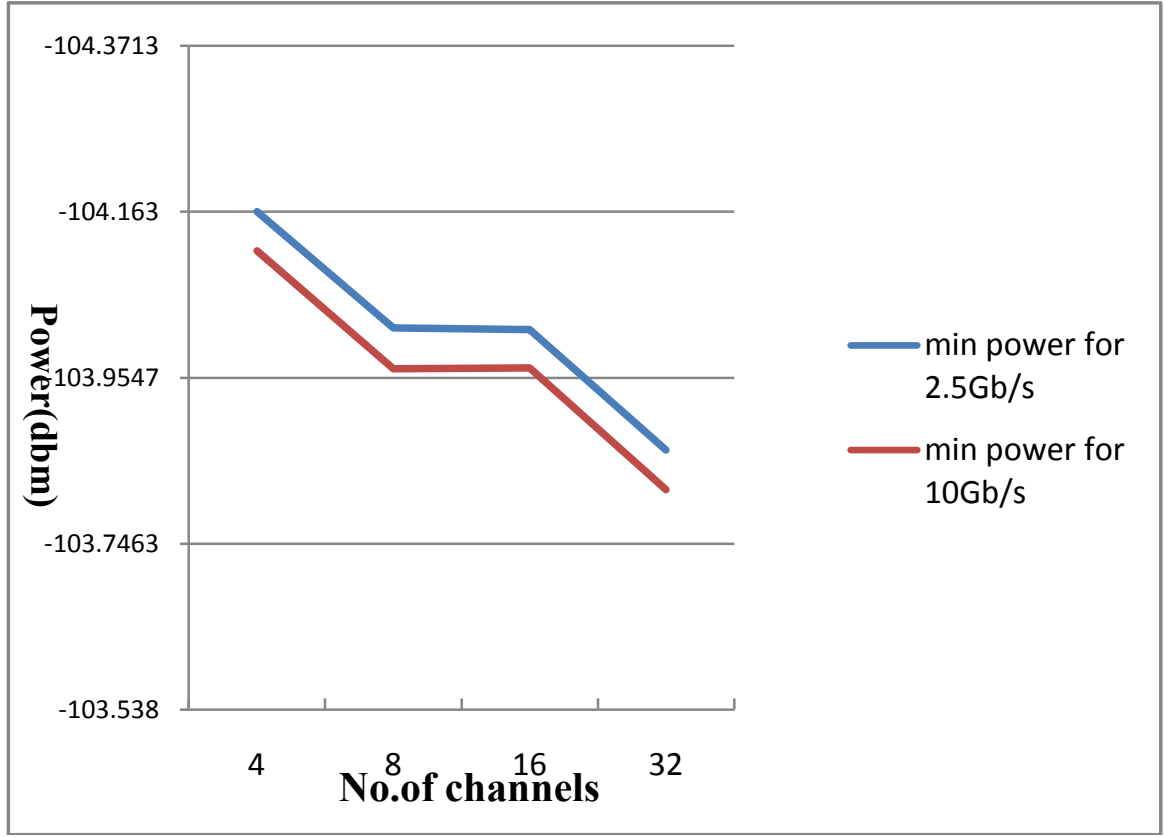

Figure 13. Relationship between minimum power and number of channels 


\section{Conclusion}

The performance analyzed of various channel spacing at different bit rates was done. In conclusion, WDM technique analyzed to utilize FBG sensor arrays for nuclear applications. Channel spacing $1 \mathrm{~nm}$ applicable to use in nuclear reactor and up to sixteen sensors at data rate $2.5 \mathrm{~Gb} / \mathrm{s}$ may used in a nuclear reactor without interference between channels. The WDM approach in this article has the potential to multiplex up to 32 FBG sensors array. With growing number of multiplexing channels of FBGs the interference effect of multi-reflection among FBG array increase, and should be taken into consideration when applying the FBGs in a single fiber to sensing the physical parameter in nuclear reactors.

\section{References}

[1] N Sangeetha, Rashi Garg, Surabhi Purwar, and Akshita Singh, "Performance Analysis of FBG DEMUX based WDM System by Varying Chirp Functions and Data Rates at Different Electrical Filters, " International Journal of Advanced Research in Computer and Communication Engineering, Vol. 3, No 3, pp. 5869-5872,Mar. 2014.

[2] Mohamed. M. Elgaud, M. S. D. Zan, A. G. Abushagur, and A. Ashrif A. Bakar, "Analysis and Simulation of Time Domain Multiplexed (TDM) Fiber Bragg Sensing Array Using OptiSystem and OptiGrating," 2016 International Conference on Advances in Electrical, Electronic and System Engineering, 14-16 Nov 2017.

[3] Alberto Fernandez Fernandez, Andrei I. Gusarov, and Francis Berghmans, "Temperature monitoring of nuclear reactor cores with multiplexed fiber Bragg grating sensors, " Opt. Eng, Vol.41, No.6, pp. 1246-1254, June 2002.

[4] Amira Zrelli, Mohamed Bouyahi, and Tahar Ezzedine, "Control and Measurement of Pressure, Temperature, and Strain Variation by Modeling Bragg Sensor," 3rd International Conference on Automation, Control, Engineering and Computer Science (ACECS'16), pp.160-165, 2016.

[5] LIU Jiansheng, ZHANG Jing, and LI Xin, "Study on Multiplexing Ability of Identical Fiber Bragg Gratings in a Single Fiber, "Chinese Journal of Aeronautics ,Vol.24, pp. 607-612, 2011. 
[6] A. Fernandez Fernandez, B. Brichard, F. Berghmans, H. El Rabii, M. Fokine, and M. Popov, "Chemical Composition Fiber Gratings in a High Mixed Gamma Neutron Radiation Field," IEEE TRANSACTIONS ON NUCLEAR SCIENCE, Vol. 53, No. 3, 2006.

[7] Andres Triana, and Daniel Pastor, "Enhancing the Multiplexing Capabilities of Sensing Networks using Spectrally Encoded Fiber Bragg Grating Sensors," Journal of Lightwave Technology, 2016.

[8] A. Fernandez Fernandez, F. Berghmans, and B. Brichard, "Multiplexed fiber Bragg grating sensors for in-core thermometry in nuclear reactors," Fiber Optic Sensor Technology II, Vol.4204, No.1, March 2001.

[9] Des Baccini, "The Effect of Gamma Radiation on Fiber Bragg Grating Sensors When Used as Radiation Dosimeters," Ph.D. Faculty of Computing, School of Engineering, Edith Cowan University, February, 2013..

[10] William Stewart, BramVanHoe, and GeertVanSteenberge, "Spectral profile tracking of multiplexed fiber Bragg grating sensors,'Optics Communications, No.357, pp. 113-119, 2015.

[11] Debabrata SIKDAR, Vinita TIWARI, and Anupam SONI, "Polarization Multiplexed Interrogation Technique for FBG Sensor Array," Photonic Sensors, Vol. 5, No. 3, pp. 193-201, 2015.

[12] Shinji Yamashita, Muneo Yokooji, "Channel spacing-tunable sampled fiber Bragg grating by linear chirp and its application to multi wavelength fiber laser," Optics Communications, Vol. 263, pp.42-46, 2006.

[13] Ningfang Song, Huizheng Yuan, Jing Jin, and Song Lin, "Spectra of Fiber Bragg Grating in Nuclear Environment," Proceedings of IC-NIDC2010.

[14] Solomon Udoh, James Njuguma, and Radhakrishna Prabhu, "Modelling and Simulation of Fiber Bragg Grating Characterization for Oil and Gas Sensing Applications," 2014 First International Conference on Systems Informatics, Modelling and Simulation. 


\section{ملخص البحث باللفة العربية}

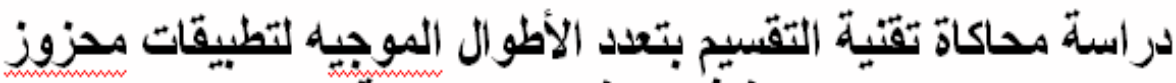 براغ في المفاعلات التووية النية}

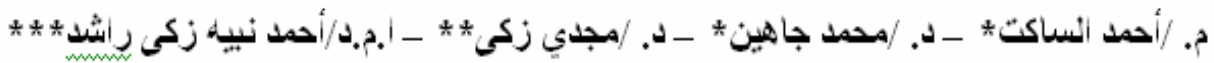

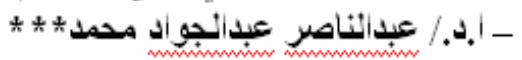

*قسم الأمان النووي و الطوارئ الإشعاعية ـ هيئه الطاقة الذرية

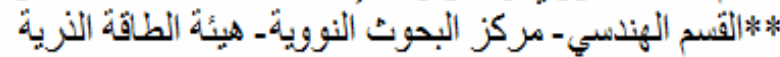

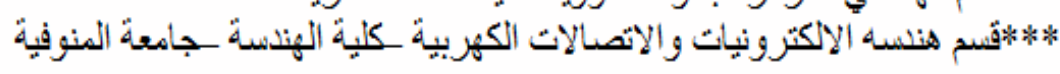

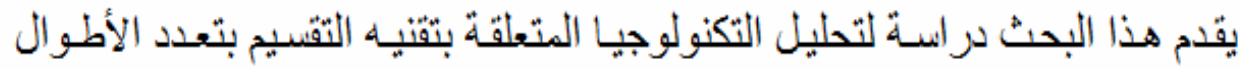

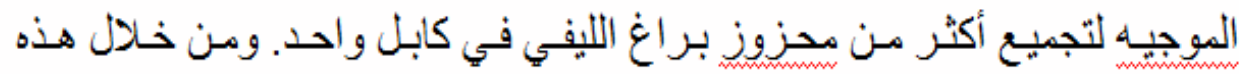

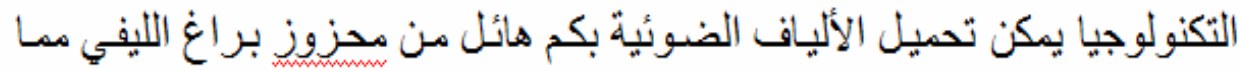

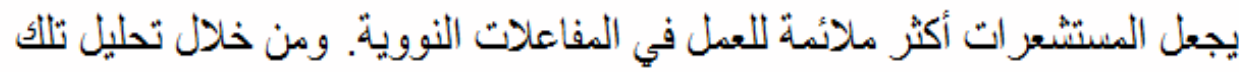

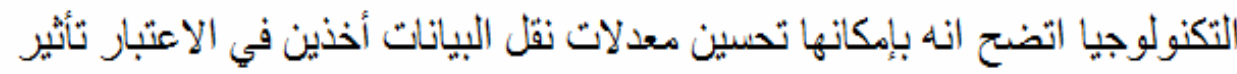

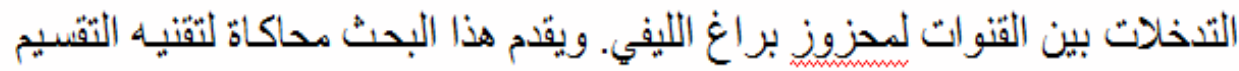

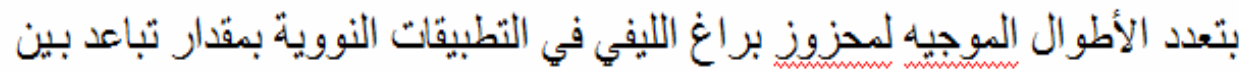

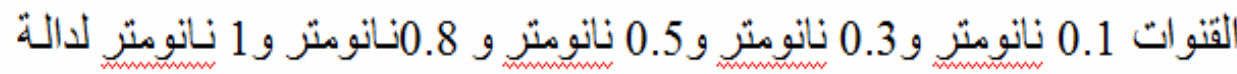

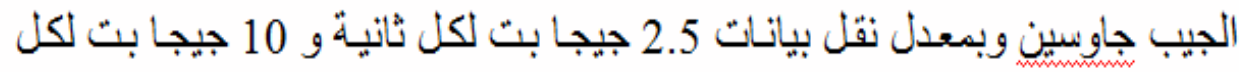

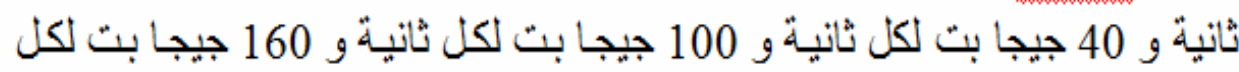

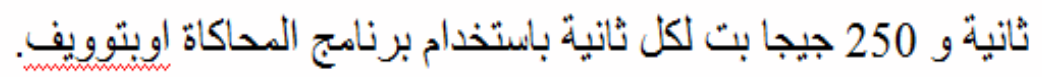

